\title{
L'Art Internet ou la médiation esthétisée : contribution à la réflexion sur l'anglais dans les filières artistiques
}

\section{Charlotte Gould}

\section{(2) OpenEdition}

\section{Journals}

Édition électronique

URL : http://journals.openedition.org/asp/1884

DOI : $10.4000 /$ asp. 1884

ISBN : 978-2-8218-0384-8

ISSN : 2108-6354

\section{Éditeur}

Groupe d'étude et de recherche en anglais de spécialité

\section{Édition imprimée}

Date de publication : 1 octobre 2001

Pagination : 115-122

ISSN : 1246-8185

Référence électronique

Charlotte Gould, "L'Art Internet ou la médiation esthétisée : contribution à la réflexion sur l'anglais dans les filières artistiques », ASp [En ligne], 31-33 | 2001, mis en ligne le 11 novembre 2010, consulté le 19 avril 2019. URL : http://journals.openedition.org/asp/1884 ; DOI : 10.4000/asp.1884

Ce document a été généré automatiquement le 19 avril 2019

Tous droits réservés 


\title{
L'Art Internet ou la médiation esthétisée : contribution à la réflexion sur l'anglais dans les filières artistiques
}

\author{
Charlotte Gould
}

1 L'inscription culturelle des études artistiques nous les fait envisager sous un angle littéraire qui se rapproche des préoccupations des anglicistes. Une telle impression de familiarité entre deux matières dessert peut-être les étudiants en art pour qui l'apprentissage de l'anglais est capital. En effet, la distance semble être un élément important dans la définition d'un domaine envisagé sous l'angle de l'anglais de spécialité. Or l'anglais artistique ressortit bien au secteur LANSAD. La spécificité de l'anglais utilisé en art doit imposer le rétablissement d'une distance d'étude qui permettra d'esquisser un système à l'usage de son enseignement.

2 L'acquisition de l'anglais est, au sein de cette filière, à la fois capitale et spécifique. Le caractère capital vient du fait que la langue d'échange et de critique du monde de l'art est l'anglais, et même un type particulier d'anglais que l'on nomme de manière orwellienne " artspeak », du fait des transformations que son maniement international lui fait subir. La spécificité, je vais tenter de l'exposer à travers l'exemple de l'utilisation qui peut être faite en cours de l'Internet. Le travail avec l'Internet est en effet exemplaire de l'idiosyncrasie de l'enseignement de l'anglais dans de telles filières. De plus, la médiation que permet cet outil de communication n'intervient que secondairement par rapport à une problématique de médiation artistique générale qu'il servira à illustrer.

3 Les potentialités artistiques de ce nouveau support s'imposent d'abord de manière évidente en termes de médiation, de diffusion d'œuvres du monde réel, mais aussi, et surtout, comme pièces créées sur la toile et qui proposent une réponse enfin radicale à un art contemporain qui cherche depuis longtemps à s'affranchir du cadre muséal. De plus, cette réalité que sont devenues les galeries virtuelles crée une nouvelle possibilité d'intervention du spectateur, donnant ainsi un double sens à la médiation technologique de l'art (mais peut-être aussi pointant le caractère galvaudé de la notion d'interactivité). 
Pourtant, du fait même que l'on se trouve encore aux balbutiements de ces pratiques d'art Internet, l'investissement d'un espace presque encore vierge s'attache, pour le moment, principalement au commentaire sur ce que nous appellerons le «médium ». L'utilisation artistique de l'Internet fait se confondre le support ou médium et le message alors que la technique rejoint la fonction esthétique. En corollaire d'une dématérialisation des supports traditionnels, c'est donc à la mise en avant de la matérialité d'un nouveau médium que l'on assiste, ainsi qu'à l'écrasement de la médiation en un support qui devient lui-même contenu.

Restera à l'avenir à situer de telles pratiques dans une chronologie de générations (à l'instar des archéologies générationnelles invoquées au sujet des artistes-vidéo) et à voir si leur succession annonce un art à venir tendant vers l'immédiateté ou bien si elle est simplement une première étape avant un dépassement du commentaire maniériste et un rétablissement de la distance médiatrice.

6 L'outil informatique Internet permet donc d'illustrer des cours par la visite de galeries virtuelles, mais l'on trouve surtout de plus en plus d'art Internet accessible en ligne ou présenté dans le cadre d'expositions. L'Internet n'a pas alors qu'un rôle de médiation de contenu. Benjamin Weil, SFMOMA curator of media arts, s'intéresse au statut particulier de ce nouveau support artistique dans sa critique en ligne « readme.text »:

Indeed, what happens online is rather unique, as it is probably the first time when the means to produce an artwork and the way it is to be distributed and experienced have come to be the same: the medium and the locus have become one. (010101.sfmoma.org, 19-11-01)

7 C'est donc parce que, en art, l'Internet n'a pas purement fonction de médiatisation que son utilisation est spécifique. Plus généralement, en art, tout support se trouve transfiguré par sa fonction artistique.

\section{« Art and Money Online » à la Tate Britain}

Quelques exemples (parmi des expérimentations diverses) d'art Internet pourront étayer cette définition de la transfiguration artistique. J'ai participé au mois de février 2001, à l'occasion d'un stage à la Tate Britain, à l'installation d'une exposition intitulée « Art and Money Online » (<www.tate.org.uk>), première exposition de groupe d'art Internet au sein de l'institution de la Tate Gallery. J'ai eu l'opportunité d'approfondir la question de la médiation artistique en travaillant auprès du personnel du musée et du critique et commissaire Julian Stallabrass qui a publié une brochure à cette occasion (2001). Depuis 1995, la Tate Gallery consacre une série d'expositions intitulée « Art Now » à l'utilisation de supports nouveaux par des artistes plutôt confidentiels. Cette dernière édition se penchait sur les relations entre art et nouvelles technologies et plus précisément sur le commentaire fait par plusieurs groupes d'artistes britanniques sur la commercialisation croissante de l'Internet.

On constate que l'orientation principale du travail artistique actuel sur la toile est fondée sur une exploration de sa structure en tant que support. La question qui se pose alors est de savoir si l'on a affaire, avec l'Internet, à un support qui a la malléabilité d'un matériau, ou à un médium. On justifiera l'emploi de ce singulier de médias (que l'on préférera à média en se référant à l'étymologie du terme et à son transit par l'anglais) car "médium" nous permet d'associer le caractère technique et médiatique de l'outil à la notion de 
support artistique, moyen terme de l'expérience esthétique, parfois traduction littérale (comme en publicité) du medium anglais.

10 Ce caractère double apparaît lorsqu'on décrit brièvement les trois œuvres présentées à Londres du 6 mars au 3 juin 2001. Le projet de Jon Thomson et Alison Craighead, CNN Interactive Just Got More Interactive (www.thomson-craighead.net) est très simple, mais son intérêt réside justement dans sa simplicité. Le site d'information en continu de la chaîne CNN est présenté presque inchangé, à l'exception de l'ajout d'une fenêtre permettant à l'internaute d'assortir les images de l'actualité de musiques d'ambiance. On clique sur festive, upbeat, dramatic, melancholy, disaster ou jubilant. Cette manipulation opère une distanciation à la fois comique et effrayante avec le site qui apparaît pour ce qu'il est : de l'infotainment.

11 La pièce présentée par le groupe Redundant Technology Initiative (RTI) deSheffield est un exemple du travail que ce groupe mène depuis plusieurs années sur la no-cost technology (< www.lowtech.org>). Dans Free Agent de vieux ordinateurs sont empilés pour former un mur d'écrans. Ils ont été récupérés gratuitement car dépassés technologiquement, et recyclés sans moyens. Tous les logiciels sont du shareware (en particulier Linux) et donc également gratuits. Chaque écran ne présente que des lignes de chiffres et de lettres dans un style qui rappelle les ordinateurs des années quatre-vingts (époque curseur). Mais juxtaposés, ils révèlent des images par transfert vidéo. On découvre le mot free sous toutes ses formes : le résultat de la recherche sur Internet de tous les sites s'y rapportant. Free est en effet la seconde requête la plus fréquente sur la toile après sex; une requête qui permet les trouvailles, comme j'ai pu le constater en aidant les artistes à télécharger tout ce que je pouvais trouver ayant trait au mot free: la philanthropie et cynophilie de ceux qui se proposent de vous offrir des économiseurs d'écran représentant des chiens, la générosité suspecte de certains fournisseurs de chocolat, en gros la possibilité de tout consommer gratuitement. La conclusion de RTI est celle du bon sens : there's no such thing as a free meal. Le "net.scape", paysage du net, saturé d'offres gratuites, cache mal son caractère commercial.

12 La dernière œuvre, celle de Lise Autogena et Joshua Portway, s'intitule Black Shoals Stock Market Planetarium (<www.blackshoals.net>). On pénètre dans une pièce sombre dans laquelle la voûte céleste semble projetée sur un hémisphère fixé au plafond. Ces images proviennent en fait d'ordinateurs reliés au réseau Internet de Reuters qui leur fournit des informations boursières. Chaque étoile représente une compagnie et leurs fusions se matérialisent en constellations plus ou moins brillantes suivant leur cotation. Il est ici sûrement prestigieux pour Reuters de se poser en sponsor (le mécénat artistique est toujours très valorisant dans le monde des affaires), mais ses dirigeants auraient peutêtre réfléchi à deux fois s'ils avaient su que Autogena et Portway allaient discrètement mais clairement moquer l'utilisation par les spéculateurs de conseils d'astrologues, faisant équivaloir information financière et prédiction pseudo-scientifique.

Cette exposition fut donc tout aussi politique que formaliste. La main-mise des intérêts capitalistes sur le développement de l'Internet y fut critiquée. C'est un aspect intéressant de ce travail, mais pas celui qui nous préoccupe ici. Ce qui frappe en fait avant tout c'est la façon dont l'art Internet explore les potentialités d'un nouveau médium en se faisant le commentaire de sa propre structure. Le site de l'artiste japonaise Tomoko Takahashi, «Word Perhect» [sic] illustre parfaitement cet attachement à la description du fonctionnement de l'outil informatique. Takahashi propose un traitement de texte personnalisé grâce auquel on peut choisir d'écrire sur un paquet de cigarettes ou au dos 
d'un ticket de métro, d'une écriture soignée ou non. Les conseils du jour qui apparaissent à chaque connexion ne concernent en rien l'informatique mais parfois la diététique. La touche « annuler la frappe » nous annonce qu'une fois que l'on a écrit quelque chose, on ne peut pas revenir en arrière, qu'il faut assumer les conséquences de ses actes. Le téléchargement se fait pendant qu'on nous exhorte à être patient : « Loading... be patient... You may have fun moving your mouse around meantime. N.B. Clicking doesn't work though!! (< www.e2.org>, 19.11.01)

La médiation est-elle alors écrasée dans un travail maniériste de mise en avant de la matérialité du support en tant que contenu? L'Internet étant à la fois instrument de médiatisation et support, la médiation artistique est-elle, lorsqu'il est utilisé, effacée par sa représentation exclusive?

\section{Rosalind Krauss et l'ère post-support}

La réflexion de Rosalind Krauss sur l'utilisation du support filmique par l'artiste belge Marcel Broodthaers dans son ouvrage A Voyage on the North Sea: Art in the Age of the PostMedium Condition (1999) peut nous permettre de réfléchir à ces questions et nous servir à analyser le statut du médium dans l'art utilisant l'Internet puisqu'elle s'attache à définir la ré-articulation post-moderne du support artistique, également médium dans le cas du cinéma et de l'Internet.

Pour Rosalind Krauss, le médium artistique ne se réduit plus aujourd'hui à ses propriétés physiques. Son approche s'oppose aux théories modernistes des années soixante et annonce l'avènement d'une ère post-support, post-médium. Quelle était la théorie moderniste concernant le support artistique avant qu'il ne se fasse médium? Tout d'abord, elle était associée à un nom, celui de Clement Greenberg. Pour Greenberg, le support (medium) était un objet physique et rien qu'un objet physique. Une approche essentialiste réduisait un tableau à son aspect plat (flatness) et à son caractère fini ( delimitation). Le modernisme aurait ainsi trouvé son apogée dans le monochrome, œuvre qui coïncide avec son origine, avec son support.

Même si cette définition attribuée à Greenberg est réductrice de sa pensée, l'importance de cette lecture historique de la définition moderniste et greenberguienne semble avoir investi la notion et avoir rendu sa ré-interprétation difficile. Le terme est chargé historiquement, ou plutôt, extrêmement déchargé par un militantisme moderniste.

Pour Krauss, le post-modernisme a donné une nouvelle place, plus importante, au support, à notre médium artistique. Il ne serait pas simple agrégat de propriétés physiques, mais structure complexe de supports techniques et de strates de conventions (des strates qui s'accumulent avec le temps et avec le développement des médias). Tout médium artistique a donc en fait une pluralité interne. Krauss parle de recursive structure (1999: 6). Une structure récursive est une structure dont certains éléments produisent les règles qui établissent cette même structure. Si le support moderniste était homogène, le médium post-moderne est hétérogène et, produisant ses propres caractéristiques, ne se définit qu'au sein de l'œuvre. Ainsi, plus l'art devient technique, moins sa médiation est affaire de technique. Selon cette définition, l'art Internet ne serait donc pas simplement supporté par un médium qui le médiatise, mais aussi constitué par ses médiums. Médiums qui s'opposeraient aux médias, même si les deux notions se complètent, le pluriel en -a étant moins courant en français qu'en anglais. Les médias sont alors technique de 
médiatisation quand des médiums rejoindraient notre notion de transfiguration esthétique. Dans une ère post-support ou post-médium, que l'on peut aussi appeler intermedia, l'œuvre perd sa spécificité technique. Voilà pourquoi on ne trouve aujourd'hui plus que des installations plutôt que les anciennes distinctions peinture, sculpture, gravure... -ce qu'on appelait les Beaux-Arts. Toute forme artistique est devenue hétérogène, elle n'est pas spécifique mais prise dans un réseau d'art. Un tableau ne peut plus être simple toile car il inclut toutes les autres dimensions spécifiques pour devenir art en général, il est aussi objet sculpture, mais aussi surtout, œuvre conceptuelle.

L'ère de la condition post-support que nous avons aussi définie comme post-médium est donc celle de l'implosion du système de pure médiation. Et pourtant, un détail constaté lors de l'installation de «Art and Money Online » aurait pu venir contredire cette théorie. M'occupant de la livraison des œuvres au musée, j'ai pu me rendre compte que des éléments arrivaient d'un peu partout, que nous en commandions beaucoup nous-même et que l'ensemble ne serait assemblé qu'in situ. De plus, toutes les pièces furent assurées commercialement et non en tant qu'œuvres d'art. Tout cela est normal, puisque chaque élément peut être remplacé en cas de panne sans dénaturer les œuvres, mais pourrait induire que les supports sont alors purement des médias : instruments de médiatisation d'un contenu artistique. Or la définition de Krauss n'exclut pas que les médiums artistiques soient des supports matériels souvent relégués au statut de pure équivalence par le principe homogénéisant de la loi du marché. L'œuvre post-moderne, post-médium, est composite et constitue un dispositif (apparatus) dans lequel les médiums artistiques ne sont pas de simples véhicules à la différence des médias, et ne se contentent pas de diffuser mais génèrent leurs propres structures. La nature artistique de l'œuvre ne se trouve pas dans un indéfinissable contenu mais bien dans un dispositif qui dans CNN Interactive Just Got More Interactive serait composé de l'information en ligne, du fait que cette information est diffusée globalement, mais aussi projetée dans le musée, de l'écran tactile, de l'image projetée, du faisceau de la projection mais aussi de la relation de ces éléments entre eux et aux spectateurs et internautes (un dispositif bien plus complexe que celui défini par Krauss pour les films de Broodthaers).

\section{Hétérogénéité du médium}

Mais si médiums et médias sont différents, les notions restent intimement liées. En effet, les possibilités d'utilisation et de signification des médiums artistiques changent avec l'évolution technique des médias. Walter Benjamin avait déjà remarqué que la « rédemption artistique » (au sens d'appropriation) d'un support technique survenait soit à sa découverte, soit au moment de son obsolescence, lorsque sa dimension utopique se libère à nouveau. Avec Internet, on se trouverait donc dans un premier moment utopique de rédemption artistique. Un point de vue esthétique qui n'empêche pas le scepticisme porté sur le médium dans « Art and Money Online ».

21 La nouveauté que l'on peut observer dans le cas de l'art Internet par rapport aux théories de Rosalind Krauss est l'introduction de la numérisation. Friedrich Kittler a commenté à ce propos dans son ouvrage Gramophone, Film, Typewriter:

The general digitalization of channels and information erases the differences among individual media. Sound and image, voice and text are reduced to surface effects, known to consumers as interface. (...) Inside the computers themselves, everything becomes a number: quantity without image, sound, or voice. And once 
optical fibre networks turn formerly distinct data flows into a standardized series of digitalized numbers, any medium can be translated into any other. With numbers, everything goes. Modulation, transformation, synchronization; delay, storage, transposition; scrambling, scanning, mapping -a total media link on a digital base will erase the very concept of medium. (1999:1-2)

totalisation et l'unification opérées par la numérisation pourraient alors menacer la finitude des médiums artistiques. Mais on remarque chez de nombreux artistes de l'Internet, comme, par exemple, chez RTI, un jeu sur l'obsolescence de la technique qui lui conserve une matérialité presque fétichiste. De plus, c'est dans les relations au sein d'un dispositif que se définissent des médiums finis. Krauss l'explique ainsi :

In order to sustain artistic practice, a medium must be a supporting structure, generative of a set of conventions, some of which, in assuming the medium itself as their subject, will be wholly « specific » to it, thus producing an experience of their own necessity. (1999: 26)

Le commentaire sur le médium ne serait alors ni écrasement de la médiation, ni totalisation de la médiatisation mais véritablement spécificité artistique de celui-ci. Plus loin, Krauss ajoute, toujours à propos du film: "The filmic apparatus presents us with a medium whose specificity is to be found in its condition as self-differing » (1999 : 44).

Ceci vaut pour l'Internet. Esthétisé et commentant sa propre structure, il ne vaut pas pour lui-même mais est en fait hétérogène et donc producteur de médiation plus que de médiatisation. C'est pourquoi on peut conclure avec Rosalind Krauss : «The specificity of mediums must be understood as differential, thus as a layering of conventions never simply collapsed into the physicality of their support » (1999: 53).

Le pendant artistique des médias serait donc les médiums. La prise en compte de ce contexte théorique d'une médiation transfigurée pourrait favoriser cette autre médiation, la réconciliation de l'enseignement de la langue et de son inscription dans une filière spécifique opérée grâce à son inscription dans le champ d'étude de l'anglais de spécialité.

\section{BIBLIOGRAPHIE}

Kittler, Friedrich. 1999. Gramophone, Film, Typewriter. Stanford : Stanford University Press.

Krauss, Rosalind. 1999. 'A Voyage on the North Sea': Art in the Age of the Post-Medium Condition. Londres : Thames and Hudson.

Stallabrass, Julian. 2001. Art and Money Online. Londres : Tate Publishing.

Bibliographie complémentaire

Lippard, Lucy. 1973. Six Years: The Dematerialization of the Art Object. Berkeley : University of California Press.

O'Brian, John (dir.). 1986. Clement Greenberg, The Collected Essays and Criticisms. Chicago : University of Chicago Press, 1986.

ASp, $31-33 \mid 2010$ 
Magnan, Nathalie et Annick Bureaud (dir.) (participation de Charlotte Gould pour la traduction). 2002. Connexions : art, réseaux, média. Paris : Éditions de l'École nationale supérieure des BeauxArts de Paris.

\section{Cyber-références}

Autogena, Lise et Joshua Portway. Consulté le 19 novembre 2001. www.blackshoals.net

Redundant Technology Initiative. Consulté le 19 novembre 2001.www.lowtech.org

Tate Gallery. Consulté le 19 novembre 2001. www.tate.org.uk

Thomson, Jon et Alison Craighead. Consulté le 19 novembre 2001. www.thomson-craighead.net

\section{RÉSUMÉS}

L'exemple de l'art Internet permet de mettre en avant la spécificité de l'approche du support artistique et la relativité de sa nomination et donc de sa traduction en fonction de son statut. En posant l'inscription théorique nécessaire et la nécessaire partialité de toute typologie esthétique, une telle analyse plaide pour la considération de l'anglais artistique comme domaine à part entière du secteur LANSAD faisant appel à une compétence double en anglais et en art dans l'optique d'un enseignement dispensé à des spécialistes plutôt qu'à l'application d'un système général d'enseignement de l'anglais à des non-spécialistes.

Of all media, the artistic medium must be apprehended in a specific way which a study of Internet art can exemplify. This specificity entails a variability in names for what we have chosen to call mediums rather than media, and, as a consequence, a variety of translations. Such an analysis, by claiming the necessary theoretical partiality of a contemporary aesthetic typology, means one favours considering English for art students as a particular subject requiring competences both in English and in art rather than the indifferent content of what is taught to non-specialists at large.

\section{INDEX}

Mots-clés : art, Internet, medium, support

\section{AUTEUR}

\section{CHARLOTTE GOULD}

Charlotte Gould est agrégée d'anglais, ancienne élève de l'École Normale Supérieure de Cachan et actuellement allocataire-monitrice à l'Université Paris 3 où elle prépare une thèse de doctorat sur le scandale dans l'art contemporain britannique des années quatre-vingt-dix.

c.gould@wanadoo.fr 